\title{
Vigor de sementes de cenoura recobertas com bioestimulante de Solieria filiformis
}

\author{
Leticia Câmara Vieira, Douglas Cristian Lucas, Mariana Bertoncini Peixoto da Silva, Flavia Bedin, Vanessa \\ Neumann Silva
}

Universidade Federal da Fronteira do Sul, UFFS. E-mail: vanessa.neumann@uffs.edu.br

\begin{abstract}
Resumo
Para garantir sementes com alta qualidade fisiológica resultando em um estande de plantas uniforme, resiliente e tolerante a estresses abióticos, procedimentos que possam melhorar o potencial da semente necessitam ser estudados. Os bioestimulantes são considerados alternativas promissoras, tanto para melhorar o desempenho fisiológico de plantas quanto na resiliência e tolerância a estresses. Objetivou-se verificar o efeito do recobrimento de sementes de diferentes cultivares de cenoura com bioestimulante a base da alga Solieria filiformis na germinação de sementes e desenvolvimento de plântulas em estresse térmico. O experimento foi conduzido em delineamento experimental inteiramente casualizado, com esquema fatorial $3 \times 4$ (temperaturas e doses), com cinco repetições. Utilizou-se sementes de cenoura das cultivares Brasília, Danvers, Esplanada e Planalto, e o recobrimento das sementes foi realizado com as doses de 0, 2, 4 e $8 \mathrm{~mL}^{-1}{ }^{-1}$ de bioestimulante. Após o recobrimento as sementes foram submetidas as seguintes avaliações: porcentagem e velocidade de germinação, comprimento de plântulas e massa seca de plântulas. Os resultados obtidos foram submetidos à análise de variância e à análise de regressão (doses) e teste de Tukey (temperaturas). A análise e interpretação dos resultados obtidos no presente trabalho permitiram concluir houve estresse térmico elevado, para todas as cultivares, na temperatura de $35^{\circ} \mathrm{C}$, resultando na redução das variáveis analisadas. Ademais, o recobrimento de sementes de cenoura com o bioestimulante de Solieria filiformis, de um modo geral, não promoveu melhorias na germinação de sementes e no desenvolvimento de plântulas de cenoura em estresse térmico $\left(35^{\circ} \mathrm{C}\right)$; no entanto, apresentou resultados benéficos para as variáveis analisadas em 20 e $30^{\circ} \mathrm{C}$.
\end{abstract}

Palavras-chave: Daucus carota; alga vermelha; estresse térmico.

Vigor of carrot seeds covered with Solieria filiformis biostimulant

\begin{abstract}
To guarantee seeds with high physiological quality performing in a uniform, resilient and tolerant to abiotic stress plant stand, procedures that can improve the potential of the processed seed. Biostimulants are considered promising alternatives, both to improve the physiological performance of plants and in resilience and stress tolerance. The objective of this study was to verify the effect of coating seeds of different cultivars of carrots with biostimulant based on algae Solieria filiformis on seed germination and seedling development under thermal stress. The experiment was conducted in a completely randomized design, with a $3 \times 4$ factorial scheme (temperatures and doses), with five replications. Carrot seeds from the cultivars Brasília, Danvers, Esplanada and Planalto were used, and the seeds were coated with doses of 0,2 , 4 and $8 \mathrm{~mL} . \mathrm{L}^{-1}$ of biostimulant. After covering, the seeds were submitted to the following: percentage and speed of germination, length of seedlings and dry mass of seedlings. The results obtained were found in the analysis of variation and in the analysis of regression (doses) and Tukey's test (moderate). The analysis and interpretation of the results obtained in the present study allowed to conclude the high thermal stress, for all varieties, at a temperature of $35^{\circ} \mathrm{C}$, to reduce the reduction of the analyzed variables. Furthermore, the covering of carrot seeds with the biostimulant of Solieria filiformis, in general, does not promote improvements in seed germination and in the development of carrot seedlings under thermal stress $\left(35^{\circ} \mathrm{C}\right)$; however, we present beneficial results for the variables analyzed at 20 and $30^{\circ} \mathrm{C}$.
\end{abstract}

Keywords: Daucus carota, red seaweed, thermal stress. 


\section{Introdução}

A cenoura (Daucus carota) pertence à família Apiaceae. Dados históricos indicam que o centro de origem da espécie é na Ásia Central (BARANSKI et al., 2012), sendo uma olerícola de grande importância econômica no país, atingindo a produção de 728.196 toneladas em 2016 em uma área de 22.254 hectares (BRASIL, 2017).

Visto que o cultivo de cenoura é realizado através de semeadura direta, para se obter um estande de plântulas uniforme, com alta produtividade e padronização do produto final é necessário o uso se sementes com alta qualidade fisiológica e sanitária (MIRANDA et al., 2017). Nesse sentido, o vigor de sementes torna-se fundamental. De acordo com a Associação Internacional de Análise de Sementes (ISTA) o vigor de sementes pode ser definido como a soma das características da semente que irão determinar o seu desempenho e potencial durante a germinação e emergência de plântulas aceitáveis em diferentes ambientes (ISTA, 2015).

Ainda, segundo Marcos Filho (2015) o vigor de sementes é resultado da interação de atributos como velocidade de germinação, crescimento de mudas, capacidade de germinar em temperaturas desfavoráveis e tolerância a estresses que irão determinar o desempenho após a semeadura. $\mathrm{O}$ autor relata que para avaliar o vigor são realizados testes para verificar o estado metabólico das sementes, como o teste de condutividade elétrica, tetrazólio e testes de avaliação de crescimento de plântulas; bem como testes para determinar a tolerância a estresses.

Uma das condições que pode gerar estresse e prejudicar a germinação e o estabelecimento de plantas de cenoura é a elevação das temperaturas. Para ser possível o cultivo de cenoura no Brasil, foram realizados diversos trabalhos de melhoramento genético através da seleção de cultivares tolerantes a temperaturas elevadas e resistentes as principais doenças (PEREIRA et al., 2007). Dentre as cultivares selecionadas para cultivo em estações mais quentes estão as cultivares do grupo Brasília. No entanto, estas cultivares não apresentam tolerância a temperaturas acima de $35^{\circ} \mathrm{C}$ (SILVA et al., 2011).

Estresses por temperaturas interrompem o processo normal de crescimento de desenvolvimento vegetal, principalmente nos estádios iniciais, levando a grandes perdas de rendimento (JAN, 2017). De acordo com Vieira et. al. (1999) para que ocorra uma rápida germinação e um bom stand de plantas de cenoura a temperatura deve estar entre 20 e $30^{\circ} \mathrm{C}$.

Uma possiblidade para mitigar o estresse causado por elevadas temperaturas é o uso de tratamentos com bioestimulantes. Segundo Vieira e Castro (2001) a mistura de dois ou mais reguladores de crescimento ou de reguladores com outras substâncias, é denominado bioestimulante. De acordo com Van Oosten et al. (2017) tratamentos com bioestimulantes apresentam-se promissores para melhorar o desempenho fisiológico de plantas e na resiliência e tolerância a estresses, como foi verificado no tratamento de sementes de salsa com $A$. nodosum reduzindo o tempo necessário para a emissão da raiz em situação de estresse térmico $\left(30^{\circ} \mathrm{C}\right)$ (SORGATTO; SILVA, 2018).

Pesquisas recentes relatam a presença de carragenanas nas paredes celulares de algas vermelhas (Rhodophyta), que podem atuar como promotoras de crescimento de vegetal modificando processos fisiológicos e bioquímicos, como por exemplo, ativando enzimas sintetizadoras, elevando os teores de clorofila, divisão celular, fotossíntese líquida, altura e biomassa de plantas, assimilação de nitrogênio e fixação de carbono (SHUKLA et al., 2016).

Ademais, de acordo com Torres et al. (2018) o efeito promotor do uso de algas vermelhas está provavelmente relacionado à presença de agaran, um polissacarídeo hidrocoloide presente em algas desse grupo. Contudo, são escassas as informações sobre o potencial do tratamento de sementes com bioestimulantes, principalmente em hortaliças.

Dessa forma, o presente trabalho foi realizado com o objetivo de verificar o efeito do recobrimento de sementes de diferentes cultivares de cenoura com bioestimulante a base da alga Solieria filiformis na germinação de sementes e desenvolvimento de plântulas em estresse térmico.

\section{Materiais e métodos}

A pesquisa foi realizada em laboratório, na Universidade Federal da Fronteira Sul, campus Chapecó, e foram utilizadas sementes de cenoura das cultivares Brasília, Danvers, Esplanada e Planalto. O delineamento experimental adotado foi inteiramente casualizado com esquema fatorial $3 \times 4$ (temperaturas e doses), com cinco repetições, separadamente para cada cultivar.

$O$ recobrimento das sementes de 
cenoura foi realizado com as doses de 0, 2, 4 e 8 $\mathrm{mL} . \mathrm{L}^{-1}$ de bioestimulante a base de Solieria filiformis composto por $7,5 \%$ de $\mathrm{Mn}$ e $13 \%$ de $\mathrm{S}$, com densidade de $1,3 \mathrm{~g} / \mathrm{cm}^{3}$; as sementes foram distribuídas em placas de Petri e adicionou-se calda preparada com os tratamentos, fazendo-se a distribuição e homogeneização; na sequência, as sementes, nos recipientes, foram deixadas sob bancada do laboratório, em ar ambiente, para que ocorresse a secagem natural por 48 horas. Após esse processo foi realizada a assepsia das sementes, com solução de álcool $70 \%$ a 30 segundos e hipoclorito de sódio $1 \%$ durante 3 minutos (BRASIL, 2009a); em seguida as sementes foram lavadas com água destilada e secadas naturalmente. Após, foram realizados os testes de germinação e crescimento de plântulas, conforme metodologia descrita a seguir.

Teste de germinação: cinco repetições de 50 sementes foram distribuídas sob duas folhas de mata borrão previamente umedecido com água destilada equivalente a 2,5 vezes o peso do papel seco, em caixas plásticas transparentes para germinação. Após esse processo, as sementes foram submetidas as temperaturas de $20^{\circ} \mathrm{C}, 30^{\circ} \mathrm{C}$ e $35^{\circ} \mathrm{C}$ em câmaras de germinação. Foram realizadas duas contagens aos sete e 14 dias após a semeadura (DAS) de acordo com os critérios a descritos em Regras para Análise de Sementes (BRASIL, 2009b).

Índice de velocidade de germinação: foram realizadas contagens diárias de sementes germinadas, durante o teste de germinação, e o índice calculado de acordo com a fórmula proposta por Maguire (1962).

Comprimento de plântulas: aos sete e 14 DAS foram coletadas 10 plântulas, de cada repetição de cada tratamento, e mensuradas com régua graduada, expressando-se os resultados em cm. plântula ${ }^{-1}$ (NAKAGAWA, 1999).

Massa seca de plântulas: após a determinação do comprimento de plântulas, as mesmas foram colocadas em sacos de papel e submetidas a secagem em estufa com circulação de ar forçado, a $65^{\circ} \mathrm{C}$ durante 72 horas e posteriormente realizada a sua pesagem em $\mathrm{mg}$ por repetição total em balança digital de precisão (NAKAGAWA, 1999).

Os resultados obtidos durante 0 experimento foram submetidos a análise de variância e se significativo para o teste $F$ foram submetidos a comparação de médias pelo teste de Tukey para o fator temperatura e análise de regressão $(p<0,05)$ pelo programa estatístico SISVAR para cada cultivar isoladamente.

\section{Resultados e Discussão}

Observou-se diferença significativa para o fator temperatura quanto a germinação e a velocidade de germinação, para as cultivares Brasília, Danvers, Esplanada e Planalto (tabela 1). As cultivares analisadas apresentaram desempenho superior em todas as variáveis nas temperaturas de 20 e $30^{\circ} \mathrm{C}$, com estresse térmico elevado, para todas as cultivares, na temperatura de $35^{\circ} \mathrm{C}$.

O baixo desempenho das cultivares na temperatura de $35^{\circ} \mathrm{C}$ pode ser explicado pelos efeitos negativos causados pelo estresse térmico nas propriedades morfo-fisiológicas principalmente no crescimento inicial de plantas, reduzindo $o$ desenvolvimento de plântulas normais, retardando processos bioquímicos e fisiológicos (JAN, 2017). De acordo com Nijabat et al. (2020) o estresse térmico causa danos a membrana celular, resultando em desnaturação de proteínas da membrana, inativando enzimas e alterando a permeabilidade e integridade da membrana reduzindo a viabilidade celular e, consequentemente, inibindo o crescimento das plantas.

De acordo com Bolton et al. (2019) a alta temperatura causa estresse térmico e é considerado um dos principais fatores abióticos que limitam a produção de cenoura devido aos seus efeitos adversos na germinação das sementes e estabelecimento de plântulas, sendo a tolerância ao calor uma característica complexa, que varia com a gravidade do estresse e o estádio de desenvolvimento das plantas. 
Tabela 1. Valores médios de germinação (G) e índice de velocidade de germinação (IVG), de sementes de cenoura, cultivares Brasília, Danvers, Esplanada e Planalto, tratadas com diferentes doses de Solieria filiformis, e submetidas a diferentes temperaturas de germinação.

\begin{tabular}{|c|c|c|c|c|c|}
\hline \multirow{4}{*}{$\begin{array}{c}\text { Temperatura } \\
\left({ }^{\circ} \mathrm{C}\right)\end{array}$} & \multicolumn{4}{|c|}{ Doses $\left(m L . L^{-1}\right)$} & \multirow[t]{4}{*}{ CV (\%) } \\
\hline & 0 & 2 & 4 & 8 & \\
\hline & \multicolumn{4}{|c|}{ Cultivar Brasília } & \\
\hline & \multicolumn{4}{|c|}{ G (\%) } & \\
\hline 20 & $75,6 a A^{*}$ & $71,2 \mathrm{aA}$ & $75,6 \mathrm{aA}$ & $73,6 \mathrm{aA}$ & \\
\hline 30 & 66,0 bA & $63,2 \mathrm{bA}$ & 67,6 aA & $70,0 \mathrm{aA}$ & 11,9 \\
\hline \multirow[t]{2}{*}{35} & $0,0 \mathrm{cA}$ & $0,0 \mathrm{cA}$ & $0,0 \mathrm{bA}$ & $0,0 \mathrm{bA}$ & \\
\hline & \multicolumn{4}{|c|}{ IVG } & \\
\hline 20 & $56,57 \mathrm{aA}$ & $54,62 \mathrm{aA}$ & $54,77 \mathrm{aA}$ & $50,22 \mathrm{aA}$ & 14,2 \\
\hline 30 & 38,92 bB & $38,49 \mathrm{bB}$ & $45,45 \mathrm{bAB}$ & $48,49 \mathrm{bA}$ & \\
\hline \multirow[t]{3}{*}{35} & $0,00 \mathrm{cA}$ & $2,98 \mathrm{cA}$ & $0,60 \mathrm{cA}$ & $0,28 \mathrm{cA}$ & \\
\hline & \multicolumn{4}{|c|}{ Cultivar Danvers } & \\
\hline & \multicolumn{4}{|c|}{$\mathrm{G}(\%)$} & \\
\hline 20 & $89,2 \mathrm{aA}^{*}$ & $88,0 \mathrm{aA}$ & $84,0 \mathrm{aA}$ & 90,0 aA & \\
\hline 30 & 86,0 aA & 90,8 aA & 86,4 aA & 87,6 aA & 8,9 \\
\hline 35 & $0,00 \mathrm{bA}$ & $0,4 \mathrm{bA}$ & $0,0 \mathrm{bA}$ & $0,0 \mathrm{bA}$ & \\
\hline \multicolumn{6}{|c|}{ IVG } \\
\hline 20 & 61,89 aA & $54,96 a A B$ & $50,55 \mathrm{aB}$ & $56,18 \mathrm{aAB}$ & 14,3 \\
\hline 30 & $50,22 \mathrm{aA}$ & $52,34 \mathrm{aA}$ & $53,08 \mathrm{aA}$ & 53,26 aA & \\
\hline 35 & $0,0 \mathrm{bA}$ & $0,22 \mathrm{bA}$ & $0,0 \mathrm{bA}$ & $0,0 \mathrm{bA}$ & \\
\hline \multicolumn{6}{|c|}{ Cultivar Esplanada } \\
\hline \multicolumn{6}{|c|}{ G (\%) } \\
\hline 20 & $67,60 \mathrm{aB}^{*}$ & $78,80 \mathrm{aA}$ & $76,80 \mathrm{aA}$ & $76,00 \mathrm{aAB}$ & \\
\hline 30 & $78,00 \mathrm{aA}$ & $62,00 \mathrm{aB}$ & $70,00 \mathrm{aAB}$ & 75,80 aA & 10,8 \\
\hline 35 & $0,00 \mathrm{bA}$ & $0,00 \mathrm{bA}$ & $0,00 \mathrm{bA}$ & $0,00 \mathrm{bA}$ & \\
\hline \multicolumn{6}{|c|}{ IVG } \\
\hline 20 & 40,13 aA & 46,03 aA & 45,78 aA & $44,77 \mathrm{aA}$ & 14,05 \\
\hline 30 & $47,40 \mathrm{aA}$ & $34,82 \mathrm{aB}$ & 41,65 aAB & $47,58 \mathrm{aA}$ & \\
\hline 35 & $0,00 \mathrm{bB}$ & $6,79 \mathrm{bAB}$ & $1,85 \mathrm{bAB}$ & 8,98 bA & \\
\hline \multicolumn{6}{|c|}{ Cultivar Planalto } \\
\hline \multicolumn{6}{|c|}{ G (\%) } \\
\hline 20 & $72,40 \mathrm{aA}^{*}$ & 77,60 aA & 73,60 aA & 69,60 aA & \\
\hline 30 & $69,60 \mathrm{aA}$ & $65,60 \mathrm{aA}$ & $71,20 \mathrm{aA}$ & $69,20 \mathrm{aA}$ & 11,5 \\
\hline 35 & $62,00 \mathrm{bA}$ & $62,00 \mathrm{bA}$ & $60,80 \mathrm{bA}$ & $55,20 \mathrm{bA}$ & \\
\hline \multicolumn{6}{|c|}{ IVG } \\
\hline 20 & $46,47 \mathrm{aA}$ & $53,81 \mathrm{aA}$ & $47,26 \mathrm{aA}$ & $47,98 \mathrm{aA}$ & 10,16 \\
\hline 30 & 53,09 aA & $44,10 \mathrm{aB}$ & $55,97 \mathrm{aA}$ & $44,79 \mathrm{aB}$ & \\
\hline 35 & $33,26 \mathrm{bA}$ & $35,45 \mathrm{bA}$ & 33,03 aA & $28,71 \mathrm{aA}$ & \\
\hline
\end{tabular}

* Médias seguidas de mesma letra minúscula na coluna e maiúscula na linha não diferem entre si pelo teste de Tukey $(p<0,05)$, separadamente para cada cultivar.

Quanto ao fator doses de bioestimulante, não foram observadas diferenças significativas entre doses e interação entre os fatores, para a variável de germinação para as cultivares Brasília, Danvers e Planalto (tabela 1); já para a cultivar Esplanada, houve efeito positivo do tratamento, nas doses de 2 e $4 \mathrm{~mL}^{-L}{ }^{1}$, comparativamente a testemunha, para sementes submetidas a temperatura de $20^{\circ} \mathrm{C}$, na variável germinação. Para a cultivar Planalto as doses de 4 e $8 \mathrm{~mL}$.L${ }^{1}$ apresentaram efeito positivo igualmente ao tratamento testemunha. Em situação de estresse térmico, a dose de $8 \mathrm{~mL}^{-L_{-}}{ }^{1}$ apresentou efeito benéfico na velocidade de germinação para a cultivar Esplanada e em $30^{\circ} \mathrm{C}$ para a cultivar Brasília. Os efeitos benéficos observados, em 
algumas doses, para algumas cultivares, podem estar relacionados a presença de carragenanas nas paredes celulares de algas vermelhas (Rhodophyta); segundo Shukla et al. (2016) esses compostos podem atuar como promotores de crescimento de vegetal, como por exemplo, ativando enzimas sintetizadoras e também na divisão celular, processos fisiológicos importantes durante a germinação de sementes e crescimento de plântulas.

Quanto ao crescimento de plântulas, observou-se efeito significativo das temperaturas de germinação para o comprimento de raízes, em todas as cultivares avaliadas, com redução drástica na temperatura de $35^{\circ} \mathrm{C}$, embora, a temperatura de $30^{\circ} \mathrm{C}$ também tenha causado efeito similar, nas sementes da cultivar Brasília, no tratamento testemunha, e na dose de $2 \mathrm{~mL}^{\mathrm{L}} \mathrm{L}^{-1}$ aos sete DAS (Tabela 2). É importante mencionar que todas as cultivares estudadas são recomendadas para cultivo no verão, e temperaturas de 30 a $35^{\circ} \mathrm{C}$ são de frequência comum, em muitas regiões do Brasil, onde se cultiva cenoura, sendo esse portanto um problema que pode prejudicar o estabelecimento de plantas a campo.

Em relação ao efeito das doses de bioestimulantes, não se obteve significância na análise de regressão, sendo apresentada comparação de médias, no desdobramento de doses dentro de cada nível de temperatura de germinação; de forma geral, não foram observados efeitos positivos dos tratamentos utilizados, tendo-se apenas resultado benéfico nas doses de 4 e $8 \mathrm{~mL}^{-1} \mathrm{~L}^{-1}$, nas temperaturas de 20 e $30 C^{\circ}$, para as variáveis comprimento de raízes aos 14 e sete DAS, respectivamente (Tabela 2). Torres et al. (2018) observou efeitos significativos no tratamento de plântulas de alface com extratos de Gracilaria caudata e Gracilaria domingensis (ambas algas vermelhas) promovendo aumento do comprimento radicular. 
Tabela 2. Valores médios de comprimento de raízes (CR) de plântulas de cenoura, avaliados aos sete (CR7) e 14 (CR14) DAS, obtidas de sementes das cultivares Brasília, Danvers, Esplanada e Planalto, tratadas com diferentes doses de Solieria filiformis, e submetidas a diferentes temperaturas de germinação.

\begin{tabular}{|c|c|c|c|c|c|}
\hline \multirow{4}{*}{$\begin{array}{c}\text { Temperatura } \\
\left({ }^{\circ} \mathrm{C}\right)\end{array}$} & \multicolumn{4}{|c|}{ Doses $\left(\mathrm{mL}^{-\mathrm{L}^{-1}}\right)$} & \multirow[t]{4}{*}{ CV (\%) } \\
\hline & 0 & 2 & 4 & 8 & \\
\hline & \multicolumn{4}{|c|}{ Cultivar Brasília } & \\
\hline & \multicolumn{4}{|c|}{$\mathrm{CR7}(\mathrm{cm})$} & \\
\hline 20 & $2,42 \mathrm{aA}$ & $2,34 \mathrm{aA}$ & $2,42 \mathrm{aA}$ & $2,58 \mathrm{aA}$ & \multirow[t]{3}{*}{22,1} \\
\hline 30 & $0,0 \mathrm{bB}$ & $0,0 \mathrm{bB}$ & $0,0 \mathrm{bB}$ & $1,19 \mathrm{bA}$ & \\
\hline 35 & $0,00 \mathrm{cA}$ & $0,00 \mathrm{cA}$ & $0,00 \mathrm{cA}$ & $0,00 \mathrm{cA}$ & \\
\hline \multicolumn{6}{|c|}{ CR14 $(\mathrm{cm})$} \\
\hline 20 & $3,69 \mathrm{aB}$ & $3,36 \mathrm{aB}$ & $4,37 \mathrm{aA}$ & $3,29 \mathrm{aB}$ & 19,8 \\
\hline 30 & $2,24 \mathrm{bA}$ & $2,26 \mathrm{bA}$ & $2,0 \mathrm{bA}$ & $2,28 \mathrm{bA}$ & \\
\hline 35 & $0,00 \mathrm{cA}$ & $0,00 \mathrm{cA}$ & $0,00 \mathrm{cA}$ & $0,00 \mathrm{cA}$ & \\
\hline \multicolumn{6}{|c|}{ Cultivar Danvers } \\
\hline \multicolumn{6}{|c|}{ CR7 $(\mathrm{cm})$} \\
\hline 20 & $1,46 \mathrm{aA}$ & 1,15 aA & $1,02 \mathrm{aA}$ & $1,35 \mathrm{aA}$ & \\
\hline 30 & 1,43 aA & 1,43 aA & $1,31 \mathrm{aA}$ & $1,38 \mathrm{aA}$ & 31,7 \\
\hline 35 & $0,0 \mathrm{bA}$ & $0,0 \mathrm{bA}$ & $0,0 \mathrm{bA}$ & $0,0 \mathrm{bA}$ & \\
\hline \multicolumn{6}{|c|}{ CR14 $(\mathrm{cm})$} \\
\hline 20 & $3,08 \mathrm{aA}$ & 2,86 aA & 3,35 aA & $2,82 \mathrm{aA}$ & 19,4 \\
\hline 30 & $2,45 \mathrm{bA}$ & $2,44 \mathrm{bA}$ & $2,32 \mathrm{bA}$ & $2,21 \mathrm{bA}$ & \\
\hline 35 & $0,0 \mathrm{cA}$ & $0,0 \mathrm{cA}$ & $0,0 \mathrm{cA}$ & $0,0 \mathrm{cA}$ & \\
\hline \multicolumn{6}{|c|}{ Cultivar Esplanada } \\
\hline \multicolumn{6}{|c|}{ CR7 $(\mathrm{cm})$} \\
\hline 20 & $1,84 \mathrm{aA}$ & $0,96 \mathrm{aB}$ & $1,72 \mathrm{aA}$ & $1,20 \mathrm{aB}$ & \\
\hline 30 & $1,19 \mathrm{bA}$ & $0,00 \mathrm{bB}$ & $0,00 \mathrm{bB}$ & $1,49 \mathrm{bA}$ & 36,3 \\
\hline 35 & $0,00 \mathrm{cA}$ & $0,00 \mathrm{cA}$ & $0,00 \mathrm{cA}$ & $0,00 \mathrm{cA}$ & \\
\hline \multicolumn{6}{|c|}{ CR14 (cm) } \\
\hline 20 & $4,36 \mathrm{aA}$ & $3,87 \mathrm{aA}$ & $3,74 \mathrm{aA}$ & $3,85 \mathrm{aA}$ & 19,5 \\
\hline 30 & $2,42 \mathrm{bA}$ & $1,90 \mathrm{bAB}$ & $1,49 \mathrm{bB}$ & $2,17 \mathrm{bA}$ & \\
\hline 35 & $0,00 \mathrm{cA}$ & $0,00 \mathrm{cA}$ & $0,00 \mathrm{cA}$ & $0,00 \mathrm{cA}$ & \\
\hline \multicolumn{6}{|c|}{ Cultivar Planalto } \\
\hline \multicolumn{6}{|c|}{ CR7 $(\mathrm{cm})$} \\
\hline 20 & $2,39 \mathrm{aA}$ & $2,77 \mathrm{aA}$ & $2,60 \mathrm{aA}$ & $2,33 \mathrm{aA}$ & \\
\hline 30 & $2,83 \mathrm{bA}$ & $3,27 \mathrm{bA}$ & $2,83 \mathrm{bA}$ & $2,79 \mathrm{bA}$ & 24,3 \\
\hline 35 & $0,00 \mathrm{cA}$ & $0,00 \mathrm{cA}$ & $0,00 \mathrm{cA}$ & $0,00 \mathrm{cA}$ & \\
\hline \multicolumn{6}{|c|}{ CR14 (cm) } \\
\hline 20 & $4,29 \mathrm{aA}$ & $4,90 \mathrm{aA}$ & $4,99 \mathrm{aA}$ & $4,86 \mathrm{aA}$ & 23,9 \\
\hline 30 & $3,41 \mathrm{bA}$ & $3,79 \mathrm{bA}$ & $2,85 \mathrm{bA}$ & $3,65 \mathrm{bA}$ & \\
\hline 35 & $0,20 \mathrm{cA}$ & $0,31 \mathrm{cA}$ & $0,27 \mathrm{cA}$ & $0,00 \mathrm{cA}$ & \\
\hline
\end{tabular}

* Médias seguidas de mesma letra minúscula na coluna e maiúscula na linha não diferem entre si pelo teste de Tukey $(p<0,05)$, separadamente para cada cultivar.

Quanto ao crescimento de parte aérea de plântulas, observou-se efeito significativo da temperatura de germinação, assim como verificado para as variáveis de germinação e comprimento de raízes, com reduções no desempenho das sementes, da cultivar Brasília, em $30^{\circ} \mathrm{C}$, e em todas as cultivares a $35^{\circ} \mathrm{C}$ (Tabela 3). Sobre o efeito de doses de bioestimulante, de maneira geral, não houve benefícios pelo tratamento das sementes, apresentando-se superior a testemunha apenas na cultivar Brasília, as doses de $8 \mathrm{~mL}^{-L^{-1}}$, na temperatura de $30^{\circ} \mathrm{C}$ aos sete DAS, e de $2 \mathrm{ml}^{.-1}$, na temperatura de $30^{\circ} \mathrm{C}$ aos 14DAS; ainda, observou-se efeitos positivos dos tratamentos com 2 e 8 mL.L $\mathrm{L}^{-1}$, comparativamente a testemunha, quando as sementes foram submetidas a temperatura de $20^{\circ} \mathrm{C}$ (tabela 3). 
Tabela 3. Valores médios de comprimento de parte aérea (CPA) de plântulas de cenoura, avaliados aos sete (CPA7) e 14 (CPA14) DAS, obtidas de sementes das cultivares Brasília, Danvers, Esplanada e Planalto, tratadas com diferentes doses de Solieria filiformis, e submetidas a diferentes temperaturas de germinação.

\begin{tabular}{|c|c|c|c|c|c|}
\hline \multirow{4}{*}{$\begin{array}{c}\text { Temperatura } \\
\left({ }^{\circ} \mathrm{C}\right)\end{array}$} & \multicolumn{4}{|c|}{ Doses $\left(\mathrm{mL}^{-\mathrm{L}^{-1}}\right)$} & \multirow[t]{3}{*}{ CV (\%) } \\
\hline & 0 & 2 & 4 & 8 & \\
\hline & \multicolumn{4}{|c|}{ Cultivar Brasília } & \\
\hline & \multicolumn{4}{|c|}{ CPA7 (cm) } & \\
\hline 20 & $4,27 \mathrm{aA}$ & $3,92 \mathrm{aAB}$ & $4,03 \mathrm{aA}$ & $3,65 \mathrm{aB}$ & \\
\hline 30 & $0,0 \mathrm{bB}$ & $0,0 \mathrm{bB}$ & $0,0 \mathrm{bB}$ & $1,64 \mathrm{bA}$ & 15,2 \\
\hline 35 & $0,00 \mathrm{cA}$ & $0,00 \mathrm{cA}$ & $0,00 \mathrm{cA}$ & $0,00 \mathrm{cA}$ & \\
\hline \multicolumn{6}{|c|}{$\mathrm{CPA} 14(\mathrm{~cm})$} \\
\hline 20 & $5,11 \mathrm{aB}$ & 5,59 aA & $4,59 \mathrm{aB}$ & $6,05 \mathrm{aA}$ & 13,3 \\
\hline 30 & $3,51 \mathrm{bB}$ & 4,38 bA & $3,24 \mathrm{bB}$ & $3,39 \mathrm{bB}$ & \\
\hline 35 & $0,00 \mathrm{cA}$ & $0,00 \mathrm{cA}$ & $0,00 \mathrm{cA}$ & $0,00 \mathrm{cA}$ & \\
\hline \multicolumn{6}{|c|}{ Cultivar Danvers } \\
\hline \multicolumn{6}{|c|}{ CPA7 $(\mathrm{cm})$} \\
\hline 20 & 3,03 aA & $2,40 \mathrm{aB}$ & $2,61 \mathrm{aAB}$ & $2,74 \mathrm{aAB}$ & \\
\hline 30 & $1,58 \mathrm{bB}$ & 2,22 bA & $1,43 \mathrm{bB}$ & $1,72 \mathrm{bAB}$ & 20,9 \\
\hline 35 & $0,0 \mathrm{cA}$ & $0,0 \mathrm{cA}$ & $0,0 \mathrm{cA}$ & $0,0 \mathrm{cA}$ & \\
\hline \multicolumn{6}{|c|}{ CPA14 $(\mathrm{cm})$} \\
\hline 20 & $5,14 \mathrm{aB}$ & 5,57 aA & $5,02 \mathrm{aB}$ & 5,57 aA & 8,7 \\
\hline 30 & $2,17 \mathrm{bB}$ & 3,34 bA & $1,89 \mathrm{bB}$ & $3,26 \mathrm{bA}$ & \\
\hline 35 & $0,0 \mathrm{cA}$ & $0,0 \mathrm{cA}$ & $0,0 \mathrm{cA}$ & $0,0 \mathrm{cA}$ & \\
\hline \multicolumn{6}{|c|}{ Cultivar Esplanada } \\
\hline \multicolumn{6}{|c|}{ CPA7 $(\mathrm{cm})$} \\
\hline 20 & $2,84 \mathrm{aA}$ & $2,75 a A$ & $2,13 \mathrm{aB}$ & $2,68 \mathrm{aA}$ & \\
\hline 30 & $1,37 \mathrm{bA}$ & $0,00 \mathrm{bB}$ & $0,00 \mathrm{bB}$ & $1,89 \mathrm{bA}$ & 28,4 \\
\hline 35 & $0,00 \mathrm{cA}$ & 0,0 OcA & $0,00 \mathrm{cA}$ & $0,00 \mathrm{cA}$ & \\
\hline \multicolumn{6}{|c|}{ CPA14 $(\mathrm{cm})$} \\
\hline 20 & $6,87 \mathrm{aA}$ & $6,24 \mathrm{aAB}$ & 6,75 aA & $6,02 \mathrm{aB}$ & 12,2 \\
\hline 30 & $3,41 \mathrm{bAB}$ & $2,89 \mathrm{bB}$ & $3,11 \mathrm{bAB}$ & $3,57 \mathrm{bA}$ & \\
\hline 35 & $0,00 \mathrm{cA}$ & $0,00 \mathrm{cA}$ & $0,00 \mathrm{cA}$ & $0,00 \mathrm{cA}$ & \\
\hline \multicolumn{6}{|c|}{ Cultivar Planalto } \\
\hline \multicolumn{6}{|c|}{ CPA7 (cm) } \\
\hline 20 & $3,03 \mathrm{aB}$ & $3,54 \mathrm{aA}$ & $3,13 \mathrm{aAB}$ & $2,95 \mathrm{aB}$ & \\
\hline 30 & $3,22 \mathrm{aB}$ & $2,85 \mathrm{aB}$ & $3,72 \mathrm{aA}$ & $2,94 \mathrm{aB}$ & 11,6 \\
\hline 35 & $0,00 \mathrm{bA}$ & $0,00 \mathrm{bA}$ & $0,00 \mathrm{bA}$ & $0,00 \mathrm{bA}$ & \\
\hline \multicolumn{6}{|c|}{ CPA14 $(\mathrm{cm})$} \\
\hline 20 & $5,75 \mathrm{aAB}$ & $5,19 \mathrm{aB}$ & 6,45 aA & $5,49 \mathrm{aB}$ & 15,6 \\
\hline 30 & 4,97 bA & $4,60 \mathrm{bA}$ & $4,83 \mathrm{bA}$ & $5,30 \mathrm{bA}$ & \\
\hline 35 & $0,33 \mathrm{cA}$ & $0,39 \mathrm{cA}$ & $0,34 \mathrm{cA}$ & $0,00 \mathrm{cA}$ & \\
\hline
\end{tabular}

* Médias seguidas de mesma letra minúscula na coluna e maiúscula na linha não diferem entre si pelo teste de Tukey $(p<0,05)$, separadamente para cada cultivar.

Quanto ao acúmulo de massa seca, observou-se efeito significativo da temperatura de germinação, assim como verificado nas demais variáveis, verificando-se desempenhos inferiores em $30^{\circ} \mathrm{C}$, e estresse térmico elevado em todas as cultivares a $35^{\circ} \mathrm{C}$ (Tabela 4). Sobre o efeito de doses de bioestimulante, de maneira geral, não foram observados efeitos benéficos pelo tratamento das sementes, apresentando-se superior a testemunha a cultivar Brasília para a variável massa seca de raiz na temperatura ideal e a Esplanada para a variável massa seca de parte aérea em 20 e $30^{\circ} \mathrm{C}$, nas doses de 2 , 4 e $8 \mathrm{~mL}^{\circ} \mathrm{L}^{-1}$, respectivamente. De maneira geral, as doses de 2 e $8 \mathrm{~mL} . \mathrm{L}^{-1}$ apresentaram efeito benéfico para as variáveis de massa seca de raiz e parte aérea para as cultivares Danvers e Esplanada em 20 e $30^{\circ} \mathrm{C}$; ainda, observou-se efeitos positivos do 
tratamento com $2 \mathrm{~mL} . \mathrm{L}^{-1}$ para massa seca de raiz, comparativamente a testemunha, quando as sementes da cultivar Planalto foram submetidas a temperatura de $30^{\circ} \mathrm{C}$ (tabela 3). Torres et al. (2018) também observaram aumento de massa de parte aérea de plantulas de alface com extratos enriquecidos com polissacarídeos da alga vermelha Gigartina Pistillata. Isso pode ser explicado pois o efeito benéfico da utilização do extrato de algas depende de muitos componentes que podem apresentar efeitos diversos em diferentes concentrações (PAULERT et al., 2010; RATHORE et al., 2009).

Tabela 4. Valores médios de massa seca de raízes (MSR) e de parte aérea (MSPA) de plântulas de cenoura, avaliados aos sete (CPA7) e 14 (CPA14) DAS, obtidas de sementes das cultivares Brasília, Danvers, Esplanada e Planalto, tratadas com diferentes doses de Solieria filiformis, e submetidas a diferentes temperaturas de germinação.

\begin{tabular}{|c|c|c|c|c|c|}
\hline \multirow{4}{*}{$\begin{array}{c}\text { Temperatura } \\
\left({ }^{\circ} \mathrm{C}\right)\end{array}$} & \multicolumn{4}{|c|}{ Doses (mL. $\mathrm{L}^{-1}$ ) } & \multirow[t]{3}{*}{$\mathrm{CV}(\%)$} \\
\hline & 0 & 2 & 4 & 8 & \\
\hline & \multicolumn{4}{|c|}{ Cultivar Brasília } & \\
\hline & \multicolumn{4}{|c|}{ MSR (mg) } & \\
\hline 20 & $0,00 \mathrm{aB}$ & 10,0 aA & 10,0 aA & 10,0 aA & \\
\hline 30 & $0,00 \mathrm{bB}$ & $0,00 \mathrm{bB}$ & $10,0 \mathrm{bA}$ & 10,0 bA & 0,0 \\
\hline 35 & $0,00 \mathrm{cA}$ & $0,00 \mathrm{cA}$ & $0,00 \mathrm{cA}$ & $0,00 \mathrm{cA}$ & \\
\hline \multicolumn{6}{|c|}{ MSPA(mg) } \\
\hline 20 & $70,00 \mathrm{aB}$ & $50,00 \mathrm{aC}$ & $70,00 \mathrm{aB}$ & $80,00 \mathrm{aA}$ & 21,7 \\
\hline 30 & $40,00 \mathrm{bB}$ & $60,00 \mathrm{bA}$ & $40,00 \mathrm{bB}$ & $40,00 \mathrm{bB}$ & \\
\hline 35 & $0,00 \mathrm{cA}$ & $0,00 \mathrm{cA}$ & $0,00 \mathrm{cA}$ & $0,00 \mathrm{cA}$ & \\
\hline \multicolumn{6}{|c|}{ Cultivar Danvers } \\
\hline \multicolumn{6}{|c|}{ MSR (mg) } \\
\hline 20 & $0,00 \mathrm{aB}$ & $10,00 \mathrm{aA}$ & $0,00 \mathrm{aB}$ & $0,00 \mathrm{aB}$ & \\
\hline 30 & $0,00 \mathrm{bB}$ & $10,00 \mathrm{bA}$ & $0,00 \mathrm{bB}$ & $10,00 \mathrm{bA}$ & 0,0 \\
\hline 35 & $0,00 \mathrm{cA}$ & $0,00 \mathrm{cA}$ & $0,00 \mathrm{cA}$ & $0,00 \mathrm{cA}$ & \\
\hline \multicolumn{6}{|c|}{ MSPA(mg) } \\
\hline 20 & $60,00 \mathrm{aB}$ & $70,00 \mathrm{aA}$ & $70,00 \mathrm{aA}$ & $70,00 \mathrm{aA}$ & 15,0 \\
\hline 30 & $20,00 \mathrm{bB}$ & $30,00 \mathrm{bA}$ & $30,00 \mathrm{bA}$ & $30,00 \mathrm{bA}$ & \\
\hline 35 & $0,00 \mathrm{cA}$ & $0,00 \mathrm{cA}$ & $0,00 \mathrm{cA}$ & $0,00 \mathrm{cA}$ & \\
\hline \multicolumn{6}{|c|}{ Cultivar Esplanada } \\
\hline \multicolumn{6}{|c|}{$\mathrm{MSR}(\mathrm{mg})$} \\
\hline 20 & 11,0 aA & $10,0 \mathrm{aB}$ & $7,0 \mathrm{aC}$ & $10,0 \mathrm{aB}$ & 0,0 \\
\hline 30 & $18,0 \mathrm{bA}$ & $9,0 \mathrm{bD}$ & $12,0 \mathrm{bc}$ & $14,0 \mathrm{bB}$ & \\
\hline 35 & $0,00 \mathrm{cA}$ & $0,00 \mathrm{cA}$ & $0,00 \mathrm{cA}$ & $0,00 \mathrm{cA}$ & \\
\hline \multicolumn{6}{|c|}{ MSPA(mg) } \\
\hline 20 & 7,20 aA & $8,80 \mathrm{bA}$ & $6,60 \mathrm{bA}$ & $7,60 \mathrm{bA}$ & 38,3 \\
\hline 30 & $5,00 \mathrm{bA}$ & $5,40 \mathrm{bA}$ & $5,00 \mathrm{bA}$ & $5,60 \mathrm{bA}$ & \\
\hline 35 & $0,00 \mathrm{cA}$ & $0,00 \mathrm{cA}$ & $0,00 \mathrm{cA}$ & $0,00 \mathrm{cA}$ & \\
\hline \multicolumn{6}{|c|}{ Cultivar Planalto } \\
\hline \multicolumn{6}{|c|}{ MSR (mg) } \\
\hline 20 & 30,0 aA & $10,0 \mathrm{aC}$ & $20,0 \mathrm{aB}$ & $20,0 \mathrm{aB}$ & 0,0 \\
\hline 30 & $20,0 \mathrm{bA}$ & $20,0 \mathrm{bA}$ & $10,0 \mathrm{bB}$ & 10,0 bB & \\
\hline 35 & $0,00 \mathrm{cA}$ & $0,00 \mathrm{cA}$ & $0,00 \mathrm{cA}$ & $0,00 \mathrm{cA}$ & \\
\hline \multicolumn{6}{|c|}{ MSPA(mg) } \\
\hline 20 & 4,00 aA & $4,00 \mathrm{aA}$ & 4,00 aA & $4,00 \mathrm{aA}$ & \\
\hline 30 & $3,00 \mathrm{bA}$ & $3,00 \mathrm{bA}$ & $3,00 \mathrm{bA}$ & $3,00 \mathrm{bA}$ & \\
\hline 35 & $0,00 \mathrm{cA}$ & $0,00 \mathrm{cA}$ & $0,00 \mathrm{cA}$ & $0,00 \mathrm{cA}$ & \\
\hline
\end{tabular}

* Médias seguidas de mesma letra minúscula na coluna e maiúscula na linha não diferem entre si pelo teste de Tukey $(p<0,05)$, separadamente para cada cultivar. 
Diante de todos resultados observados nessa pesquisa, verifica-se que o extrato de Solieria filiformis pode ser um importante bioestimulante na cultura da cenoura na temperatura ideal e em $30^{\circ} \mathrm{C}$, apresentando efeitos significativos referente ao vigor de sementes e crescimento de plântulas. Dutta et al. (2019) também observou melhoras significativas no índice de vigor e massa das plântulas em sementes de pimenta tratadas com as algas Kappaphycys alvarezii e Gracilaria eduli. A temperatura é um fator que influencia diretamente a velocidade e porcentagem de germinação; e nesse caso, a baixa emergência de plântulas em sementes submetidas ao estresse térmico $\left(35^{\circ} \mathrm{C}\right)$ ocasionou reduções em todas as variáveis analisadas.

No entanto, novas pesquisas com outras dosagens e cultivares são necessárias para obterse mais informações sobre o potencial de uso do bioestimulante para tratamento de sementes de cenoura, visando aumento do vigor de sementes, desenvolvimento das plântulas e a capacidade de resistência à estresse térmico.

\section{Conclusão}

O recobrimento de sementes de cenoura com o bioestimulante de Solieria filiformis não promove melhorias na germinação de sementes e no desenvolvimento de plântulas de cenoura em estresse térmico $\left(35^{\circ} \mathrm{C}\right)$. No entanto, observou-se resultados benéficos para as variáveis de germinação e velocidade de germinação, crescimento de plântula e acúmulo de massa seca na temperatura ideal e em $30^{\circ} \mathrm{C}$. Analisando-se o efeito das temperaturas, observa-se estresse térmico elevado, para todas as cultivares, na temperatura de $35^{\circ} \mathrm{C}$; o estresse por alta temperatura também afetou as demais variáveis analisadas.

\section{Referências}

BARANSKI, R.; MAKSYLEWICZ-KAUL, A.; NOTHNAGEL, T.; CAVAGNARO, P.F.; SIMON, P.W.; GRZEBELUS, D. Diversidade genética de cultivares de cenoura (Daucus carota L.) revelada pela análise de loci SSR. Genetic Resources and Crop Evolution, v.59, 163-170, 2012. https://doi.org/10.1007/s10722$\underline{011-9777-3}$
BOLTON, A.; NIJABAT, A.; MAHMOOD-URREHMAN, M.; NAVEED, N.H.; MANNAN, A.T.M. ALI, A.; SIMON, P. Variation for heat tolerance during seed germination in diverse carrot [Daucus carota (L.)] Germplasm. Hortscience, v.54, n.9, p.1470-1476, 2019. https://doi.org/10.21273/HORTSCl14144-19.

BRASIL. Confederação da Agricultura e Pecuária do Brasil. Mapeamento e qualificação da cadeira produtiva das hortaliças do Brasil. Brasília: CAN, 2017. 79p.

BRASIL. Ministério da Agricultura, Pecuária e Abastecimento. Manual de Análise Sanitária de Sementes. Ministério da Agricultura, Pecuária e Abastecimento. Secretaria de Defesa Agropecuária. Brasília, DF: Mapa/ACS, 2009a. 200p.

BRASIL. Ministério da Agricultura, Pecuária e Abastecimento. Regras para análise de sementes. Ministério da Agricultura, Pecuária e Abastecimento. Secretaria de Defesa Agropecuária. Brasília, DF: Mapa/ACS, 2009b. $395 p$.

DUTTA, S.K.; LAYEK, J.; AKOIJAM, R.S.; $\quad$ BOOPATHI, T.; VANLALHMANGAIHA, S.S.; SINGH, S.B.; LUNGMUANA, P.N. Seaweed extract as natural priming agent for augmenting seed quality traits and yield in Capsicum frutescens L. Journal of Applied Phycology, v.31, p.3803-3813, 2019.

ISTA. International rules for seed testing. Basserdorf, Switzerland: International Seed Testing Association, 2015. https://doi.org/10.1007/s10811-019-01871-0

JAN, S.A. Impact of salt drought, heat and frost stresses on morphobiochemical and physiological properties of Brassica species: An updated review. Journal of Rural Development and Agriculture, v.2, n.1, p. 1-10, 2017.

MAGUIRE, J.D. Speeds of germination-aid selection and evaluation for seedling emergence and vigor. Crop Science, v.2, p. 176-177, 1962. https://doi.org/10.2135/cropsci1962.0011183X00 $\underline{0200020033 x}$

MARCOS FILHO, J. Seed vigor testing: an overview 
of the past, present and future perspective: an overview of the past, present and future perspective. Scientia Agricola, v.72, n.4, p.363374, 2015. http://dx.doi.org/10.1590/0103-90162015-0007

MIRANDA, R.M.; DIAS, D.C.F.S.; PICOLI, E.A.T.; SILVA, P.P.; NASCIMENTO, W.M. Physiological quality, anatomy and histochemistry during the development of carrot seeds (Daucus carota L.). Ciência e Agrotecnologia, v.41, n.2, p.169-180, $2017 . \quad$ http://dx.doi.org/10.1590/141370542017412009216.

NAKAGAW, A. J. Testes de vigor baseados no desempenho de plântulas. In: KRZYZANOWSKI, F.C.; VIEIRA, R.D.; FRANÇA NETO, J.B. Vigor de Sementes: conceitos e testes. Londrina: ABRATES, 1999. 218p.

NIJABAT, A.; BOLTON, A.; MAHMOOD-URREHMAN, M.; SHAH, A. I.; HUSSAIN, R.; NAVEED, N. H.; ALI, A.; SIMON, P. Cell Membrane Stability and Relative Cell Injury in Response to Heat Stress during Early and Late Seedling Stages of Diverse Carrot (Daucus carota Germplasm. Hortscience, v.55, n. 9, p. 1446-1452, set. 2020. http://dx.doi.org/10.21273/hortsci15058-20 .

PAULERT, R.; $\quad$ TALAMINI, V.; CASSOLATO, J.E.F.; DUARTE, M.E.R.; NOSEDA, M.D.; SMANIA JR, A.; STADNIK, M.J. Effects of sulfated polysaccharide and alcoholic extracts from green seaweed Ulva fasciata on anthracnose severity and growth of common bean (Phaseolus vulgaris L.). Journal of Plant Diseases and Protection, v.116, n.6, p.263270, 2009. https://doi.org/10.1007/BF03356321

PEREIRA, R. S.; NASCIMENTO, W. M.; VIEIRA, J. V. Germinação e vigor de sementes de cenoura sob condições de altas temperaturas. Horticultura Brasileira, v.25, n.2, p.215-219, 2007. https://doi.org/10.1590/S0102-

$\underline{05362007000200017}$

RATHORE, S.S.; CHAUDHARY, D.R;. BORICHA, G.N.; GHOSH, A.; BHATT, B.P.; ZODAPE, S.T.; PATOLIA, J.S. Effect of seaweed extract on the growth, yield and nutrient uptake of soybean (Glycine max) under rainfed conditions. South African Journal of Botany, v.75, p. 351-355, 2009. https://doi.org/10.1016/i.sajb.2008.10.009

SILVA, G. O.; VIEIRA, J.V.; NASCIMENTO, W. M. Estratégias de seleção para germinação de sementes de cenoura em altas temperaturas. Semina: Ciências Agrárias, v. 32, n. 3, p. 849-854, $2011 . \quad \quad$ http://dx.doi.org/10.5433/16790359.2011v32n3p849.

SHUKLA, P. S.; BORZA, T.; CRITCHLEY, A. T.; PRITHIVIRAJ, B. Carrageenans from Red Seaweeds As Promoters of Growth and Elicitors of Defense Response in Plants. Frontiers In Marine Science, v. 3, p. 1-9, 2016.

http://dx.doi.org/10.3389/fmars.2016.00081.

SORGATTO, K. P.; SILVA, V. N. Embebição de sementes de salsa com Ascophyllum nodosum: efeitos na germinação e crescimento de plântulas sob estresse térmico: efeitos na germinação e crescimento de plântulas sob estresse térmico. Acta Biológica Catarinense, v.5, n.3, p.98-106, 2018. http://dx.doi.org/10.21726/abc.v5i3.518.

SOUZA, B. G. A.; PEREIRA, L. A. FREITAS; SOUZA, J.V.G.A.; ALBUQUERQUE, J.R.T.S.; SOUSA, L.V.; BARROS JÚNIOR, A.P. Crescimento e desenvolvimento de mudas de tomate sob efeito de extrato Ascophyllum nodosum. Revista Verde de Agroecologia e Desenvolvimento Sustentável, v.12, n.4, p.712-716, 2017. http://dx.doi.org/10.18378/rvads.v12i4.4932.

TORRES, P.; NOVAES, P.; FERREIRA, L. G.; SANTOS, J. P.; MAZEPA, E.; DUARTE, M. E. R.; NOSEDA, M. D.; CHOW, F.; SANTOS, D. Y. A.C. Effects of extracts and isolated molecules of two species of Gracilaria (Gracilariales, Rhodophyta) on early growth of lettuce. Algal Research, v.32, p.142149, 2018. http://dx.doi.org/10.1016/j.algal.2018.03.016.

VAN OOESTEN, M. J.; PEPE, O.; PASCALE, S. de; SILLETTI, S.; MAGGIO, A. The role of biostimulants and bioeffectors as alleviators of abiotic stress in crop plants. Chemical and Biological Technologies in Agriculturen, v.4, n.5, p.1-12, 2017. http://dx.doi.org/10.1186/s40538-0170089-5

VIEIRA, E.L.; CASTRO, P.R.C. Ação de bioestimulante na germinação de sementes, vigor de plântulas, crescimento radicular e produtividade de soja. Revista Brasileira 
Sementes, v.3, n.2, p.222-228, 2001.

https://doi.org/10.17801/0101-

3122/rbs.v23n2p222-228

VIEIRA, J.V.; PESSOA, H.B.S.V.; MAKISHIMA, N. A

cultura da cenoura. Embrapa Hortaliças. Brasília:

Embrapa, 1999. 77p. (Coleção plantar; 43) 